\title{
Adaptation to Brassica Host Genotypes by a Single-Spore Isolate and Population of Plasmodiophora brassicae (Clubroot)
}

J. M. LeBoldus, Department of Plant Pathology, North Dakota State University, Fargo, ND 58108, USA; V. P. Manolii, Department of Agricultural, Food and Nutritional Science, University of Alberta, Edmonton, AB T6G 2P5, Canada; T. K. Turkington, Lacombe Research Centre, Agriculture and Agri-Food Canada, Lacombe, AB T4L 1W1, Canada; and S. E. Strelkov, Department of Agricultural, Food and Nutritional Science, University of Alberta, Edmonton, AB T6G 2P5, Canada

\begin{abstract}
LeBoldus, J. M., Manolii, V. P., Turkington, T. K., and Strelkov, S. E. 2012. Adaptation to Brassica host genotypes by a single-spore isolate and population of Plasmodiophora brassicae (clubroot). Plant Dis. 96:833-838.

Plasmodiophora brassicae, the cause of clubroot of crucifers, is an increasingly important pathogen of canola (Brassica napus) in Alberta, Canada. In response, clubroot-resistant canola genotypes are being deployed to help reduce yield losses. Two experiments were conducted to examine the effect on P. brassicae virulence of repeated exposure of a population and single-spore isolate of the pathogen to the same host. The first experiment examined changes in the index of disease over five cycles of infection on seven Brassica hosts (European Clubroot Differential [ECD] 02, ECD 04, ECD 05, ECD 15, '45H26', '45H29', and $08 \mathrm{~N} 823 \mathrm{R})$. The second experiment tested the virulence of five cycled populations ('45H29', 08N823R, ECD 05, and ECD 15) and

three cycled single-spore isolates ('45H29', 08N823R, and ECD05) on four resistant canola genotypes ('73-77', '73-67', VT-SD-09, and ' $9558 \mathrm{C}$ '). The results from these experiments clearly demonstrate the ability of both single-spore isolates and populations of $P$. brassicae to rapidly erode the resistance present in the two canola genotypes, ' $45 \mathrm{H} 29$ ' and $08 \mathrm{~N} 823 \mathrm{R}$. Although the index of disease increased on these two genotypes, the four resistant canola genotypes remained resistant to all the cycled populations and single-spore isolates in the second experiment. These results underscore the importance of crop rotation in the management of clubroot in Alberta.
\end{abstract}

Plasmodiophora brassicae Woronin, an obligate parasite causing clubroot, is one of the most damaging soilborne diseases of crucifers worldwide. Historically, in Canada, clubroot has been an important disease of a variety of Brassica vegetables in the provinces of British Columbia, Ontario, Quebec, and the Maritimes (21). Recently, however, clubroot was identified on Brassica napus L. (canola) on the Canadian prairies (27). Initially, several clubrootinfested fields were found clustered around the city of Edmonton, Alberta. More extensive surveys have revealed the presence of this pathogen in hundreds of commercial fields in central Alberta and a few isolated cases in southern Alberta (6,23-25). Severe infestations can result in yield losses of 30 to $100 \%$ (12). Given the extensive area in canola production in Alberta, one-third of which is located on acidic soils that favor disease development, clubroot has become a serious concern for canola producers in this province and across the prairies.

Crop rotation has been the simplest and most effective means of managing this disease. However, this strategy may not be practical for canola producers, because $P$. brassicae resting spores are capable of remaining viable in the soil for 20 years or more (28). The application of lime to increase soil $\mathrm{pH}$ and suppress disease development has also been an effective approach for the management of clubroot $(7,12)$. However, this requires repeated application of large amounts of lime (10) and is likely not a feasible option for canola, which is typically cropped over very large numbers of hectares. Fungicide control has also been attempted but results are often inconsistent and the associated cost may be prohibitive (17). The application of calcium cyanamide is considered one of the most effective means of control but is also quite expensive (15). Given the emerging importance of $P$. brassicae as a pathogen of

Corresponding author: S. Strelkov, E-mail: stephen.strelkov@ualberta.ca

Accepted for publication 15 December 2011.

http://dx.doi.org/10.1094/PDIS-09-11-0807

(C) 2012 The American Phytopathological Society canola on the Canadian prairies, several recent studies have begun to examine a variety of management strategies which could be used over the large areas typical of canola production.

Ahmed et al. (2), for example, assessed the efficacy of bait crops to reduce the inoculum loads of $P$. brassicae resting spores under field and greenhouse conditions. They found that, under greenhouse conditions, resting spore concentrations and the corresponding disease severities were slightly reduced following two cycles of cruciferous bait crops (canola or Chinese cabbage) compared with noncruciferous crops (red clover, perennial ryegrass, orchardgrass, or bentgrass). However, bait crops had no effect on clubroot severity at two field locations where concentrations of resting spores were high (2). In a study examining the effect of seedling age on clubroot severity, Hwang et al. (13) observed a reduction in clubroot severity as the age of inoculated seedlings increased from 1 to 4 weeks, indicating the potential utility of seed treatment fungicides with a long residual period as disease management tools. In another study examining the efficacy of seven biological control agents and two fungicides at reducing clubroot severity, two biological control agents, Bacillus subtilis Ehrenberg \& Cohn and Gliocladium catenulatum Gilman \& Abbott, were found to be as effective as the fungicides fluazinam and cyazofamid under greenhouse conditions (18). Unfortunately, these biological control agents were not effective under field conditions, perhaps due to the relatively dry weather experienced that year (18). Finally, the manipulation of seeding dates has been shown to reduce disease severity in other cropping systems. Preliminary results indicated that early seeding significantly reduced the severity of clubroot at one of two field sites and increased canola yields by up to $58 \%$ (9). Although some of the findings discussed above may be incorporated into clubroot management strategies in the future, the cropping of clubroot-resistant canola cultivars remains the most desirable and cost-effective means of controlling this disease (23).

Recently, several clubroot-resistant canola cultivars have become commercially available and are currently deployed in infested fields. In many other host-pathogen systems, the deployment of resistant cultivars has resulted in the selection of increased virulence in the pathogen population, potentially reducing the durabil- 
ity and stability of resistance $(1,3,4,14)$. Although the inoculation of different host cultivars with pathotype mixtures of $P$. brassicae has been shown to shift the composition of the mixture to the more virulent pathotypes on that host $(8,14)$, the effect of clubroot resistance on specific populations and single-spore isolates of $P$. brassicae is untested. To this end, a population (SACAN03-1) and single-spore-derived isolate (SACAN03-ss1) of the pathogen, collected in Alberta and previously characterized for virulence and pathotype designation (pathotype $=3)(26,30)$, were used in a pathogen cycling experiment. Both the population and single-spore isolate were used to inoculate seven different host genotypes exhibiting a range of resistances (resistant, moderately resistant, and susceptible) in five successive cycles of inoculation, resting spore extraction, and reinoculation. The objectives of this study were twofold: (i) to evaluate the change in virulence of the population and single-spore isolate following five successive infection cycles on the same host cultivars and (ii) to assess the potential durability of resistance in the current clubroot-resistant canola cultivars.

\section{Materials and Methods}

Terminology. As previously defined by Xue et al. (30), a "population" of $P$. brassicae resting spores refers to a collection of all resting spores recovered from infested soil or clubs of an infected plant, whereas a "single-spore isolate" refers to a collection of resting spores derived from a gall produced by inoculation with a single resting spore.

Pathogen population. A single population of $P$. brassicae resting spores, SACAN03-1, and one single-spore isolate, SACAN03ss 1 , were used in the study described below. The population was originally obtained from clubbed canola roots collected near St. Albert, Alberta, Canada and characterized by Strelkov et al. (26). The single-spore isolate was derived from the SACAN03-1 population and characterized by Xue et al. (30). Single spore isolations were conducted by placing a $0.5-\mu \mathrm{l}$ drop of spore suspension on the coverslip of a microscope slide (30). The drop was examined at 100 -fold magnification to confirm the presence of a single resting spore. Upon confirmation, the roots of a 1- or 2-week-old seedling of the universally susceptible host, Chinese cabbage (Brassica rapa var. pekinensis) 'Granaat', were immersed in the droplet (30). Both the population and single-spore isolate were reproduced and maintained on Granaat.

Plant material. Changes in the virulence of the population and single-spore isolate of $P$. brassicae were evaluated following each of five successive cycles of inoculation, resting spore extraction, and reinoculation. In total, seven different hosts, four from the European Clubroot Differential (ECD) set (5) and three canola genotypes, all with known levels of resistance to P. brassicae, were inoculated separately with SACAN03-1 and SACAN03-ss1 (Table 1). Following the five inoculation cycles, the population and single-spore isolate were recharacterized on the complete ECD set (5), two additional differential hosts (B. napus L. var. napobrassica 'Laurentian' [29] and B. napus 'Brutor' [22]), and four clubrootresistant $B$. napus genotypes. Seed of the ECD hosts were obtained from Horticulture Research International, Genetic Resources Unit, Wellesbourne, Warwick, United Kingdom. Seed of Laurentian and Brutor were purchased from the Crucifer Genetics Cooperative (Madison, WI). The three canola genotypes used in the pathogen cycling experiment ('45H26', '45H29', and 08N823R) were obtained from Pioneer Hi-Bred Production Ltd. (Caledon, Ontario, Canada). Two of the four canola genotypes used in the pathotyping experiment, VT-SD-09 and '9558C', were obtained from Viterra (Calgary, Alberta, Canada) and the remaining two, '73-77' and '7367', were obtained from Monsanto (Lethbridge, Alberta, Canada).

Host inoculation. Five successive rounds of inoculation, spore extraction, and reinoculation were conducted. In the first round, resting spores of both the population SACAN03-1 and the singlespore isolate SACAN03-ss1 were extracted from frozen root tissue using a procedure modified from that of Williams (29) and previously described by Tewari et al. (27). Concentrations of P. brassicae resting spores were determined using a hemocytometer (VWR, Mississauga, Ontario, Canada) and were adjusted to a final concentration of approximately $1 \times 10^{7}$ spores $/ \mathrm{ml}$ with sterile distilled water. Seven-day-old seedlings, germinated on filter paper in petri dishes, were inoculated by dipping the roots in the spore suspension for $10 \mathrm{~s}$. Following inoculation, the seedlings were immediately planted in 6-by-6-by-6-cm plastic pots, one seedling per pot, filled with Metro-Mix 290 growth medium (Terra-Lite 2000 series; WR Grace and Company, Ajax, Ontario, Canada). The pots were thoroughly watered and transferred to a greenhouse kept at $22^{\circ} \mathrm{C}$ with a 16-h photoperiod. The potting mixture was kept saturated with water for the first week after inoculation, then watered and fertilized as required. The experimental design was a completely randomized design replicated three times. Each host-single-spore isolate or -population combination occurred 16 times per replicate. These 16 seedlings were considered to be a single experimental unit. All subsequent cycles of inoculation were conducted in an identical manner to that described above with one exception: galls collected from the previous cycle were used as the source of inoculum for the subsequent cycle. In most cases, disease development was sufficient to provide inoculum of the correct concentration for the following round of inoculations. However, in some of the inoculations with the resistant hosts ECD 02 and ECD 04, root gall development was very limited and the seedlings in the next cycle were sown into the potting mixture from the previous round. Finally, the virulence patterns of the population cycled on '45H29', 08N823R, ECD 05, or ECD 15 and the single-spore isolate cycled on ' $45 \mathrm{H} 29$ ', $08 \mathrm{~N} 823 \mathrm{R}$, or ECD 05 were evaluated on the complete ECD set (5), two additional differential B. napus hosts (Laurentian [29] and Brutor [22]), and four clubroot-resistant canola genotypes ('73-77', '73-67', VT-SD-09, and '9558C'), using galls from the fifth and final cycle as the source of inoculum. The experimental design was a completely randomized design replicated four times. In total, 16 seedlings of each host, grown in 4-by-4-by-4-cm plastic pots, were inoculated with either the single-spore isolates or populations described above. Each group of 16 seedlings was considered to be a single experimental unit. Although earlier work

Table 1. Hosts used to evaluate changes in the virulence of a population (SACAN03-1) and single-spore isolate (SACAN03-ss1) of Plasmodiophora brassicae, following five cycles of inoculation, spore extraction, and reinoculation

\begin{tabular}{|c|c|c|}
\hline Cultivar or line & ECD number ${ }^{\mathbf{a}}$ & Initial resistance \\
\hline \multicolumn{3}{|c|}{ Polish rape (Brassica rapa subsp. rapifera) } \\
\hline Line AAbbCC & 02 & Resistant \\
\hline Line AABBCC & 04 & Resistant \\
\hline \multicolumn{3}{|l|}{ Canola (B. napus) } \\
\hline $45 \mathrm{H} 29$ & na & Resistant \\
\hline 08N823R & na & Moderately resistant \\
\hline $45 \mathrm{H} 26$ & na & Susceptible \\
\hline \multicolumn{3}{|c|}{ Kale $(B$. oleracea var. capitata subvar. laciniata) } \\
\hline 'Verheul' & 15 & Moderately resistant \\
\hline \multicolumn{3}{|c|}{ Chinese cabbage (B. rapa var. perkinensis) } \\
\hline 'Granaat' & 05 & Susceptible \\
\hline
\end{tabular}

${ }^{\mathrm{a}} \mathrm{ECD}=$ European Clubroot Differential and na $=$ not applicable (there is no ECD number). 
in Europe suggested that ECD 10 ('Wilhemsburger') and Laurentian gave similar reactions to $P$. brassicae (G. Dixon, personal communication), both hosts were included in the current study, because they give distinct reactions to populations of the pathogen from Alberta (23) and other parts of North America (29).

Disease assessment. Six weeks following inoculation, the roots were removed from the growing medium, washed with water, and evaluated for clubroot symptom development. Disease severity was rated on a 0 -to-3 scale adapted from Kuginuki et al. (16), where 0 = no galling, 1 = a few small galls (small galls on less than onethird of the roots), $2=$ moderate galling (small to medium to galls on one-third to two-thirds of the roots), and $3=$ severe galling (medium to large galls on more than two-thirds of the roots). An index of disease (ID) was calculated using a formula from Horiuchi and Hori (11) as modified by Strelkov et al. (26): $I D(\%)=\{[\Sigma(n \times 0+$ $n \times 1+n \times 2+n \times 3)] / N \times 3\} \times 100 \%$, where $\Sigma$ is the sum total; $n$ is the number of plants in a class; $N$ is the total number of plants; and $0,1,2$, and 3 are the symptom severity classes. The ID value for each host and single-spore isolate or population combination was calculated for each experimental unit (16 seedlings). Mean ID was calculated by averaging the ID for each host across repetitions.
Statistical analysis. SAS was used to conduct a one-way analysis of variance for each cultivar-isolate (single-spore isolate or population) combination, and Tukey's highly significant difference was used to determine if there were significant differences $(\alpha=$ 0.05 ) in the mean ID among cycles for each combination (20). Isolates and populations were classified into pathotypes by calculating the mean ID and its associated $95 \%$ confidence interval (CI) for each genotype-isolate combination. Genotypes were considered resistant if the mean ID was less than $50 \%$ and the $95 \%$ CI did not overlap 50\%. A pathotype designation was then assigned as per the differential systems of Williams (29), Buczacki et al. (5), and Somé et al. (22).

\section{Results}

Changes in $P$. brassicae virulence over five successive infection cycles. There were no significant differences among ID values for the single-spore isolate SACAN03-ss1 or the population SACAN03-1 among the five cycles of inoculation on the resistant $B$. rapa genotypes ECD 02 and ECD 04 (Fig. 1). In all four cases, the ID value remained very low, never rising above $1.4 \%$ in any one cycle. In the case of the universally susceptible cultivar, ECD 05,

\section{SINGLE-SPORE}

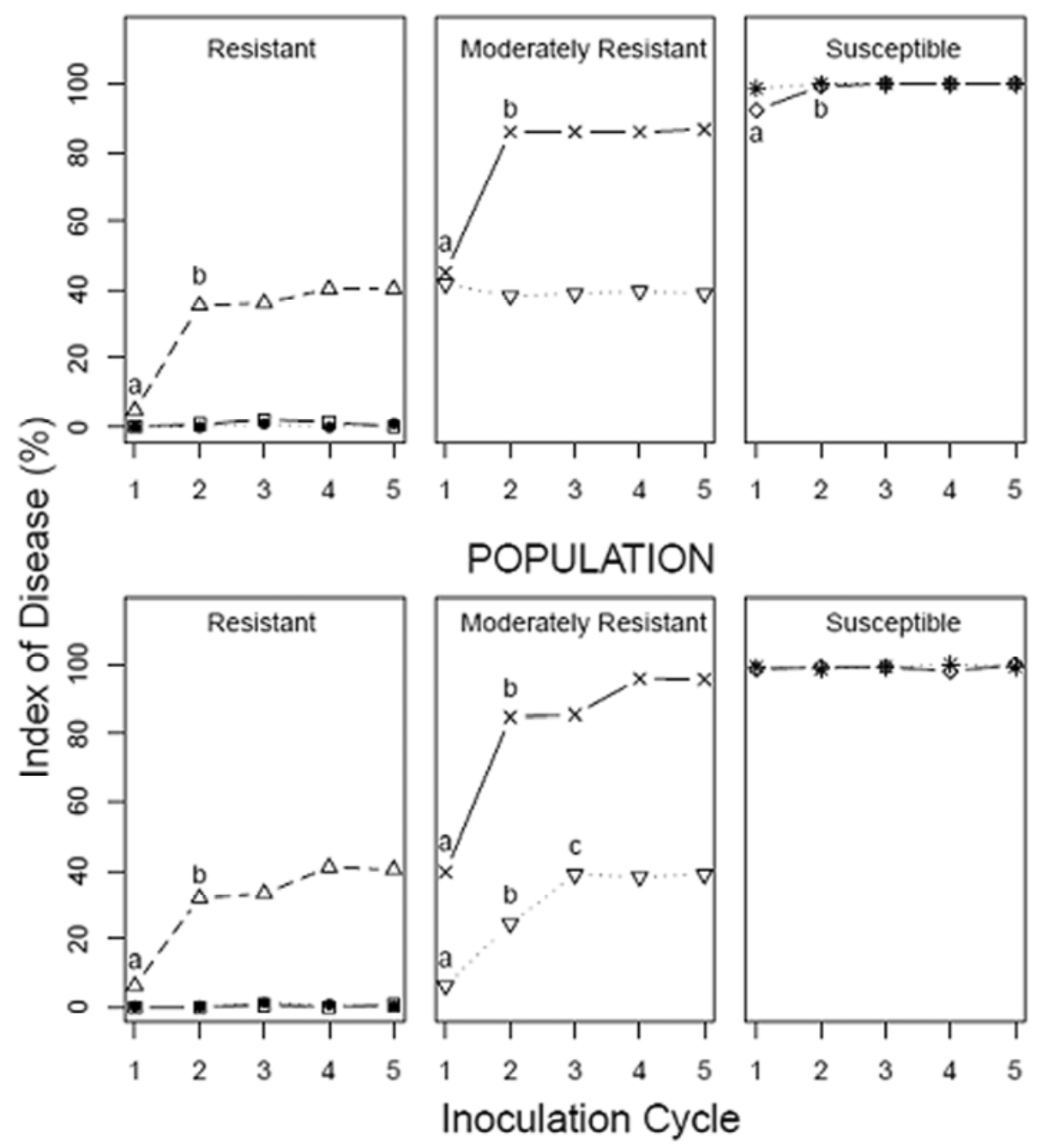

a ECD $02 \bullet$ ECD $04 \triangle 45 \mathrm{H} 29 \times 08 \mathrm{~N} 823 \mathrm{R} \nabla \mathrm{ECD} 15 \diamond 45 \mathrm{H} 26 \quad *$ ECD 05

Fig. 1. Change in mean index of disease (ID) following five cycles of inoculation, isolation, and reinoculation on the same host with a single-spore isolate (SACAN03-ss1) or population (SACAN03-1) of Plasmodiophora brassicae. In total, seven different hosts exhibiting different levels of clubroot resistance were inoculated (resistant = European Clubroot Differential [ECD] 02, ECD 04, and '45H29'; moderately resistant = 08N823R and ECD 15; and susceptible = '45H26' and ECD 05). Mean ID values with different letters are significantly different for a particular host. 
the ID value in any one inoculation cycle for both the single-spore isolate and population never dropped below $98 \%$ (Fig. 1). A similar pattern occurred for the susceptible canola genotype ' $45 \mathrm{H} 26$ '-single-spore isolate combination, for which the ID value never dropped below $98 \%$ (Fig. 1). However, in the case of the '45H26'population combination, a significant difference $(P<0.001$; Fig. 1$)$ between the first (ID $=92 \%$ ) and second $(I D=99 \%)$ cycle was observed; following this shift, the IDs remained similar for the remaining three cycles (Fig. 1). The resistant canola genotype ' $45 \mathrm{H} 29$ ' and moderately resistant genotype $08 \mathrm{~N} 823 \mathrm{R}$ also underwent significant increases in ID value (Fig. 1). In the case of '45H29', when inoculated with the population, the ID value increased from 5 to $35 \%(P<0.001$; Fig. 1$)$ and then remained relatively constant in all subsequent cycles. The ' $45 \mathrm{H} 29$ '-single-spore isolate combination followed a similar pattern, increasing from 6 to $32 \%(P<0.001$; Fig. 1$)$ between the first and second cycles and then remaining relatively constant in subsequent generations. The moderately resistant genotype $08 \mathrm{~N} 823 \mathrm{R}$ also showed a significant increase in ID from the first to second cycle for the population and single-spore isolate (Fig. 1). The increase was from 45 to $86 \%(P<$ $0.001)$ when inoculated with the population and from 40 to $85 \%(P$ $<0.001$ ) when inoculated with the single-spore isolate (Fig. 1).
ECD 15 did not show a consistent pattern for the population and single-spore isolate inoculations (Fig. 1). In the case of repeated inoculation with the single-spore isolate, there were no significant differences in ID among cycles on ECD 15 (ID = 40\%); however, in the case of repeated inoculation with the population, there was a significant difference between the first (ID $=6 \%$ ) and second cycle (ID $=24 \% ; P=0.006 ;$ Fig. 1 ) and between the second (ID $=24 \%$ ) and third cycle (ID $=38 \% ; P=0.02$; Fig. 1 ). All subsequent cycles were similar (Fig. 1).

Virulence of cycled isolates and populations on differential hosts. The pathotype designations of the single-spore isolates cycled on ' $45 \mathrm{H} 29$ ', $08 \mathrm{~N} 823 \mathrm{R}$, and ECD 05 and the populations cycled on ' $45 \mathrm{H} 29$ ', 08N823R, ECD 05, and ECD 15 were unchanged from those reported for the uncycled isolate and population $(26,30)$. In all cases, the cycled populations and single-spore isolates were classified as pathotype 3 according to Williams (29), $\mathrm{P}_{2}$ according to Somé et al. (22), or 16/15/12 according to the ECD set (5) (Table 2). These designations were identical to those described for the single-spore isolate SACAN03-ss1 by Xue et al. (30) and the population SACAN03-1 by Strelkov et al. (26).

Virulence of cycled isolates and populations on other clubroot-resistant canola genotypes. With only three exceptions, all

Table 2. Reactions of 17 Brassica differential hosts in response to inoculation with four populations and three single-spore isolates of Plasmodiophora brassicae following five cycles of repeated inoculation, spore extraction, and reinoculation on the Brassica hosts '45H29', 08N823R, ECD 05, and ECD 15'

\begin{tabular}{|c|c|c|c|c|c|c|c|}
\hline \multirow[b]{2}{*}{ Genotype $^{b}$} & \multicolumn{4}{|c|}{ Populations } & \multicolumn{3}{|c|}{ Single-spore isolates } \\
\hline & $45 \mathrm{H} 29$ & 08N823R & ECD 05 & ECD 15 & $45 \mathrm{H} 29$ & 08N823R & ECD 05 \\
\hline$\overline{\text { ECD } 01}$ & $1.9 \pm 5.5$ & $2.8 \pm 9.4$ & $5.5 \pm 9.4$ & $2.8 \pm 0.0$ & $1.9 \pm 5.5$ & $2.8 \pm 7.2$ & $6.5 \pm 7.7$ \\
\hline ECD 02 & $0.0 \pm 0.0$ & $0.0 \pm 0.0$ & $0.0 \pm 0.0$ & $0.0 \pm 0.0$ & $1.9 \pm 7.7$ & $0.9 \pm 5.5$ & $1.9 \pm 7.7$ \\
\hline ECD 03 & $10.2 \pm 7.7$ & $18.5 \pm 7.7$ & $24.1 \pm 5.5$ & $13.0 \pm 7.7$ & $19.4 \pm 7.1$ & $22.2 \pm 7.2$ & $26.9 \pm 5.5$ \\
\hline ECD 04 & $0.9 \pm 5.5$ & $2.8 \pm 7.2$ & $4.6 \pm 7.7$ & $2.8 \pm 7.2$ & $0.9 \pm 5.5$ & $1.9 \pm 7.7$ & $2.8 \pm 9.4$ \\
\hline ECD 05 ('Granaat') & $97.2 \pm 7.2$ & $98.1 \pm 5.5$ & $100.0 \pm 0.0$ & $98.1 \pm 5.5$ & $94.4 \pm 7.1$ & $96.3 \pm 5.5$ & $100.0 \pm 0.0$ \\
\hline ECD 06 ('Nevin') & $91.7 \pm 7.1$ & $94.4 \pm 7.1$ & $98.1 \pm 5.5$ & $92.6 \pm 5.4$ & $86.1 \pm 7.2$ & $89.8 \pm 5.5$ & $90.8 \pm \mathbf{5 . 5}$ \\
\hline ECD 07 & $95.4 \pm 7.7$ & $95.3 \pm 5.5$ & $100.0 \pm 0.0$ & $96.3 \pm 5.5$ & $92.6 \pm 8.8$ & $95.3 \pm 5.5$ & $98.1 \pm 7.7$ \\
\hline ECD 08 & $95.3 \pm \mathbf{5 . 5}$ & $\mathbf{9 7 . 2} \pm \mathbf{0 . 0}$ & $100.0 \pm 0.0$ & $95.4 \pm 7.7$ & $90.8 \pm 5.5$ & $93.5 \pm 5.4$ & $89.8 \pm 7.7$ \\
\hline ECD 09 & $95.4 \pm 7.7$ & $94.4 \pm 7.1$ & $99.1 \pm 5.5$ & $94.4 \pm 7.1$ & $89.8 \pm 5.5$ & $91.7 \pm 7.1$ & $94.4 \pm 0.0$ \\
\hline ECD 10 ('Wilhemsburger') & $14.8 \pm 5.5$ & $18.5 \pm 5.4$ & $22.2 \pm 7.2$ & $19.4 \pm 7.1$ & $10.2 \pm 5.5$ & $19.4 \pm 7.1$ & $22.2 \pm 7.2$ \\
\hline ECD 11 ('Badger Shipper') & $17.6 \pm 5.4$ & $19.4 \pm 9.4$ & $22.2 \pm 7.2$ & $20.4 \pm 7.7$ & $20.4 \pm 7.7$ & $25.0 \pm 7.2$ & $27.8 \pm 7.2$ \\
\hline ECD 12 & $25.0 \pm 7.2$ & $27.8 \pm 9.5$ & $35.2 \pm 5.5$ & $31.5 \pm 5.4$ & $26.9 \pm 8.9$ & $36.1 \pm 7.2$ & $40.8 \pm 5.5$ \\
\hline ECD 13 ('Jersey Queen') & $63.9 \pm 13.6$ & $63.0 \pm 8.9$ & $89.8 \pm 5.5$ & $64.8 \pm 10.4$ & $55.6 \pm 7.1$ & $64.8 \pm 7.7$ & $71.3 \pm 7.7$ \\
\hline ECD 14 & $93.5 \pm 8.8$ & $95.3 \pm 5.5$ & $97.2 \pm 7.2$ & $92.6 \pm 8.8$ & $84.3 \pm 8.9$ & $88.0 \pm 8.9$ & $91.7 \pm 0.0$ \\
\hline ECD 15 ('Verheul') & $29.7 \pm 5.5$ & $38.0 \pm 7.7$ & $42.6 \pm 5.4$ & $37.0 \pm 7.7$ & $29.7 \pm 5.5$ & $35.2 \pm 7.7$ & $38.0 \pm 5.5$ \\
\hline 'Laurentian' & $92.6 \pm 5.4$ & $96.3 \pm 5.5$ & $99.1 \pm 5.5$ & $92.6 \pm 5.4$ & $88.9 \pm 7.2$ & $91.7 \pm 7.1$ & $92.6 \pm 5.4$ \\
\hline 'Brutor' & $97.2 \pm 7.2$ & $98.1 \pm 7.7$ & $100.0 \pm 0.0$ & $97.2 \pm 0.0$ & $94.4 \pm 7.1$ & $96.3 \pm 5.5$ & $100.0 \pm 0.0$ \\
\hline Final pathotype $^{\mathrm{c}}$ & $16 / 15 / 12,3, P_{2}$ & $16 / 15 / 12,3, P_{2}$ & $16 / 15 / 12,3, P_{2}$ & $16 / 15 / 12,3, P_{2}$ & $16 / 15 / 12,3, P_{2}$ & $16 / 15 / 12,3, P_{2}$ & $16 / 15 / 12,3, P_{2}$ \\
\hline Initial pathotype ${ }^{d}$ & $16 / 15 / 12,3, P_{2}$ & $16 / 15 / 12,3, P_{2}$ & $16 / 15 / 12,3, P_{2}$ & $16 / 15 / 12,3, P_{2}$ & $\mathbf{3}, \mathbf{P}_{2}$ & $\mathbf{3}, \mathbf{P}_{2}$ & $\mathbf{3}, \mathbf{P}_{2}$ \\
\hline
\end{tabular}

${ }^{a}$ Populations and single-spore isolates were derived from SACAN03-1 and SACAN03-ss1, respectively, and were originally characterized by Strelkov et al. (25) and Xue et al. (29). Mean indices of disease (ID) in bold were considered to represent a susceptible reaction for pathotype classification purposes.

${ }^{\mathrm{b}}$ European Clubroot Differential $(\mathrm{ECD}) 01=$ Brassica rapa subsp. rapifera line aaBBCC; ECD $02=$ B. rapa subsp. rapifera line AAbbCC; ECD $03=B$. rapa subsp. rapifera line $\mathrm{AABBcc}$; $\mathrm{ECD} 04=$ B. rapa subsp. rapifera line $\mathrm{AABBCC}$; $\mathrm{ECD} 05=$ B. rapa var. pekinensis; $\mathrm{ECD} 06=$ B. napus var. napus Nevin; ECD $07=$ B. napus var. napus 'Giant Rape'; ECD $08=$ B. napus var. napus Giant Rape selection; ECD $09=$ B. napus var. napus New Zealand resistant rape; $\mathrm{ECD} 10=$ B. napus var. rapifera; $\mathrm{ECD} 11=$ B. oleracea var. capitata Badger Shipper; ECD $12=$ B. oleracea var. capitata 'Bindsachsener'; ECD $13=$ B. oleracea var. capitata Jersey Queen; ECD $14=$ B. oleracea var. capitata 'Septa'; ECD $15=$ B. oleracea var. capitata subvar. laciniata; Laurentian $=$ B. napus var. napobrassica; and Brutor $=$ B. napus.

${ }^{\mathrm{c}}$ Final pathotype designations are based on the ECD (5), Williams (28), and Somé et al. (21) differential sets. ECD designations are based on the binary notation system of nomenclature developed by Buczacki et al. (5).

${ }^{\mathrm{d}}$ Initial pathotype designations from Strelkov et al. (25), Xue et al. (29), and unpublished data (for SACAN03-1 on the differentials of Somé et al. [21]).

Table 3. Mean index of disease (ID) and 95\% confidence interval of four clubroot-resistant canola genotypes inoculated with four populations and three single-spore-isolates of Plasmodiophora brassicae following five cycles of repeated inoculation, spore extraction, and reinoculation on the Brassica hosts '45H29', 08N823R, ECD 05, and ECD 15'

\begin{tabular}{|c|c|c|c|c|c|c|c|}
\hline \multirow[b]{2}{*}{ Host genotype } & \multicolumn{4}{|c|}{ Populations } & \multicolumn{3}{|c|}{ Single-spore isolates } \\
\hline & $45 \mathrm{H} 29$ & 08N823R & ECD 05 & ECD 15 & $45 \mathrm{H} 29$ & 08N823R & ECD 05 \\
\hline $73-77$ & $5.5 \pm 9.4$ & $1.9 \pm 7.7$ & $4.6 \pm 8.9$ & $5.5 \pm 9.4$ & $0.0 \pm 0.0$ & $2.8 \pm 9.4$ & $5.5 \pm 9.4$ \\
\hline $73-67$ & $8.6 \pm 2.9$ & $0.0 \pm 0.0$ & $0.0 \pm 0.0$ & $0.0 \pm 0.0$ & $0.0 \pm 0.0$ & $0.0 \pm 0.0$ & $0.0 \pm 0.0$ \\
\hline VT-SD-09 & $1.9 \pm 7.7$ & $0.0 \pm 0.0$ & $0.0 \pm 0.0$ & $0.0 \pm 0.0$ & $0.0 \pm 0.0$ & $0.0 \pm 0.0$ & $0.0 \pm 0.0$ \\
\hline $9558 \mathrm{C}$ & $11.1 \pm 9.5$ & $0.0 \pm 0.0$ & $0.0 \pm 0.0$ & $0.0 \pm 0.0$ & $0.0 \pm 0.0$ & $0.0 \pm 0.0$ & $0.0 \pm 0.0$ \\
\hline
\end{tabular}

a Populations and single-spore isolates were derived from SACAN03-1 and SACAN03-ss1, respectively, and were originally characterized by Strelkov et al. (25) and Xue et al. (28). ECD = European Clubroot Differential. 
clubroot-resistant canola genotypes exhibited a similar pattern of either complete resistance (ID $=0.0 \%$ ) or an ID value that was not significantly different from zero (Table 3) after inoculation with the cycled isolates or populations of $P$. brassicae. However, genotypes 73-67 (ID = 8.6 $\pm 2.9 \%)$, VT-SD-09 (ID = $1.9 \pm 7.7 \%$ ), and $9558 \mathrm{C}$ (ID $=11.1 \pm 9.5 \%$ ) inoculated with the $P$. brassicae population cycled on ' $45 \mathrm{H} 29$ ' exhibited indices of disease greater than and significantly different from $0.0 \%$ (Table 3 ).

\section{Discussion}

The mean ID on both the resistant canola ' $45 \mathrm{H} 29$ ' and the moderately resistant $08 \mathrm{~N} 823 \mathrm{R}$ increased significantly after the first cycle of inoculation with the single-spore isolate or population of P. brassicae (Fig. 1). Following this initial shift, however, the ID remained relatively constant. One potential explanation for this phenomenon, at least in the case of ' $45 \mathrm{H} 29$ ', may be the presence of both major and minor genes for clubroot resistance, previously documented in Brassica spp. (19). The major gene resistance may have eroded following the first inoculation cycle, resulting in an increase in ID in the following cycle (Fig. 1). The lack of any further changes in ID may be a result of several minor resistance genes conferring partial resistance to $P$. brassicae. A similar pattern was observed for the moderately resistant variety 08N823R (Fig. 1). However, given the initial intermediate ID value observed on $08 \mathrm{~N} 823 \mathrm{R}$, it is likely that a source of resistance different from that in '45H29' was involved. The exact nature of the resistance in the commercial canola genotypes analyzed in this study is unknown because of proprietary considerations.

A second possible explanation for the shifts in ID observed after repeated cycling on the same host was initially proposed by Williams (29) and Buczacki et al. (5), who suggested that heterogeneity in the pathogen population may result in intermediate or fluctuating ID values. These authors indicated that it may be necessary to conduct two cycles of inoculation and spore extraction, without a change in ID, before a population can be considered homogeneous $(5,29)$. This is a possible explanation in the case of the ECD 15population combination, where the ID value for the population stabilized at a level similar to that of the single-spore isolate (assumed to be homogenous) following two cycles of inoculation (Fig. 1). However, this explanation is unlikely in the case of the two canola genotypes ' $45 \mathrm{H} 29$ ' and $08 \mathrm{~N} 823 \mathrm{R}$, because of the consistent pattern observed across single-spore isolates (putatively homogenous) and populations (putatively heterogeneous) (Fig. 1). It is important to note that, although the population was considered to be heterogeneous relative to the single-spore isolate, it was originally collected from a single gall. Had the experiment been conducted using a mixture of pathotypes or in a naturally infested field, the increase in pathogen virulence may have been greater due to greater diversity in the initial pathogen population.

The rapid shift in the ID values of the two canola genotypes ( $45 \mathrm{H} 29$ ' and $08 \mathrm{~N} 823 \mathrm{R}$ ) underscores the importance of crop rotation on the Canadian prairies. As a consequence of the cost and uncertainty associated with other methods of clubroot control, long crop rotations out of susceptible hosts have been emphasized for the management of this disease on large hectarage crops (23). However, the relatively high value of the canola crop, especially compared with other cropping options, in combination with the availability of clubroot-resistant varieties, increases the temptation to grow canola year after year or in very short rotation on the same field. The ID shifts observed in this study are clear evidence that this would likely reduce the length of time that a resistance source could be deployed.

It is interesting to note that the increases in ID observed in this study did not correspond to increases in IDs on any of the differential hosts inoculated with the cycled single-spore isolates or populations of $P$. brassicae (Table 2). That is, there were no corresponding changes in the pathotype designations of any of the single-spore isolates or populations cycled on different hosts. However, the population cycled on '45H29' induced ID values significantly greater than $0 \%$ on three of the four resistant canola geno- types ('73-67', VT-SD-09, and '9558C'), whereas the single-spore isolate cycled on ' $45 \mathrm{H} 29$ ' did not induce disease on any of these hosts (Table 3). This may reflect a greater adaptive potential in the inoculum originally derived from a population versus a singlespore isolate of $P$. brassicae. Nonetheless, even in those resistant genotypes for which the ID was greater than zero after inoculation with the pathogen population cycled on ' $45 \mathrm{H} 29$ ', the extent of erosion of the resistance was significantly less than on '45H29' itself. The lack of a major change in the ID on these resistant genotypes suggests the introduction of resistance genes different from those incorporated into ' $45 \mathrm{H} 29$ ' or $08 \mathrm{~N} 823 \mathrm{R}$. These results raise the possibility of rotating different clubroot-resistant canola cultivars on clubroot-infested fields; however, the durability of the resistance in these genotypes is still uncertain, whereas the benefits of crop rotation are widely documented (23).

The results from these two experiments clearly demonstrate the ability of both single-spore isolates and populations of $P$. brassicae to rapidly erode the resistance present in the two canola genotypes '45H29' and 08N823R. Although resistance in other canola genotypes appears to remain strong (Table 3), the rapid shift in ID (Fig. 1) should underscore the importance of crop rotation in the management of clubroot. Given that field populations are likely to be more diverse than the single-spore isolate or population used in this study, care must be given to where, when, and at what frequency resistant cultivars should be deployed.

\section{Acknowledgments}

We thank Pioneer Hi-Bred, Viterra, and Monsanto for generously providing seed of the various canola genotypes; the Alberta Crop Industry Development Fund, the Alberta Canola Producers Commission, SaskCanola, the Manitoba Canola Growers Association, and the Canola Council of Canada through the Agriculture and Agri-Food Canada Clubroot Risk Mitigation Initiative for their the financial support; and G. Dixon (University of Reading, UK) and an anonymous reviewer for their helpful comments on the manuscript.

\section{Literature Cited}

1. Ahmed, H. U., Hwang S. F., Gossen, B. D., Chang, K. F., Howard, R. J. Strelkov, S. E., and Turnbull, G. D. 2009. Adaptation to host (chickpea) genotype by isolates of Didymella rabiei following repeated cycles of infection. J. Plant Dis. Prot. 116:177-181.

2. Ahmed, H. U., Hwang S. F., Strelkov, S. E., Gossen, B. D., Peng, G. Howard, R. J., and Turnbull, G. D. 2011. Assessment of bait crops to reduce inoculum of clubroot (Plasmodiophora brassicae) of canola. Can. J. Plant Sci. 91:545-551.

3. Ahmed, H. U., Mundt, C. C., Hoffer, M. E., and Coakley, S. M. 1996. Selective influence of wheat cultivars on pathogenicity of Mycosphaerella graminicola (Anamorph Septoria tritici). Phytopathology 86:454-458.

4. Alexander, H. M., Groth, J. V., and Roelfs, A. P. 1985. Virulence changes in Uromyces appendiculatus after five asexual generations on a partially resistant cultivar Phaseolus vulgaris. Phytopathology 75:449-453.

5. Buczacki, S. T., Toxopeus, H., Mattusch, P., Johnston, T. D., Dixon, G. R., and Hobolth, L. A. 1975 Study of physiologic specialization in Plasmodiophora brassicae: Proposals for attempted rationalization through an international approach. Trans. Br. Mycol. Soc. 65:295-303.

6. Cao, T., Manolii, V. P., Hwang, S. F., Howard, R. J., and Strelkov, S. E. 2009. Virulence and spread of Plasmodiophora brassicae (clubroot) in Alberta, Canada. Can. J. Plant Pathol. 31:321-329.

7. Donald, C., and Porter, I. 2009. Integrated control of clubroot. J. Plant Growth Regul. 28:289-303.

8. Fahling, M., Graf, F., and Siemens, J. 2003. Pathotype separation of Plasmodiophora brassicae by host plant. J. Phytopathol. 151:425-430.

9. Gossen, B. D., McDonald, M. R., Hwang, S. F., and Kalpana, K. C. 2009 Manipulating seeding date to minimize clubroot (Plasmodiophora brassicae) damage in canola and vegetable Brassicas. (Abstr.) Phytopathology 99:S45.

10. Hildebrand, P. D., and McRae, K. D. 1998. Control of clubroot caused by Plasmodiophora brassicae with non-ionic surfactants. Can. J. Plant Pathol. 20:1-11.

11. Horiuchi, S., and Hori, M. 1980. A simple greenhouse technique for obtaining high levels of clubroot incidence. Bull. Chugoku Natl. Agric. Exp. Stn. Ser. E (Environ. Div.) 17:33-55

12. Howard, R. J., Strelkov, S. E., and Harding, M. W. 2010. Clubroot of cruciferous crops-new perspectives on an old disease. Can. J. Plant Pathol. $32: 43-57$

13. Hwang, S. F., Ahmed, H. U., Strelkov, S. E., Goosen, B. D., Turnbull, G. D., Peng, G., and Howard, R. J. 2011. Seedling age and inoculum density affect clubroot severity and seed yield in canola. Can. J. Plant Sci. 91:183-190.

14. Jones, D. R., Ingram, D. S., and Dixon, G. R. 1982. Factors affecting tests 
for differential pathogenicity in populations of Plamodiophora brassicae. Plant Pathol. 31:229-238.

15. Klasse, H. J. 1996. Calcium cyanamide-an effective tool to control clubroot-a review. Acta Hortic. 407:403-409.

16. Kuginuki, Y., Hiroaki, Y., and Hirai, M. 1999. Variation in virulence of Plasmodiophora brassicae in Japan tested with clubroot-resistant cultivars of Chinese cabbage (Brassica rapa L. spp. Perkinensis). Eur. J. Plant Pathol. 105:327-332.

17. Naiki, T., and Dixon, G. R. 1987. The effects of chemicals on developmental stages of Plasmodiophora brassicae (clubroot). Plant Pathol. 35:316-327.

18. Peng, G., McGregor, L., Lahlali, B. D., Hwang, S. F., Adhikari, K. K., Strelkov, S. E., and McDonald, M. R. 2011. Potential biological control of clubroot on canola and crucifer vegetable crops. Plant Pathol. 60:566-574.

19. Piao, Z., Ramchiary, N., and Lim, Y. P. 2009. Genetics of clubroot resistance in Brassica species. J. Plant Growth Regul. 28:252-264.

20. R Development Core Team. 2011. R: A Language and Environment for Statistical Computing. R Foundation for Statistical Computing. Vienna. www.R-project.org

21. Rimmer, S. R., Kutcher, H. R., and Morrall, R. A. A. 2003. Diseases of canola and mustard. Pages 129-146 in: Diseases of Field Crops in Canada. K. L. Bailey, B. D. Gossen, R. K. Gugel, and R. A. A. Morrall, eds. Canadian Phytopathological Society, Saskatoon, SK, Canada.

22. Somé, A., Manzanares, M. J., Laurens, F., Baron, F., Thomas, G., and Rouxel, F.1996. Variation for virulence on Brassica napus L. amongst Plasmodiophora brassicae collections from France derived from single-spore isolates. Plant Pathol. 45:432-439.
23. Strelkov, S. E., Hwang, S. F., Howard, R. J., Hartman, M., and Turkington, T. K. 2011. Progress towards the sustainable management of clubroot (Plas modiophora brassicae) of canola on the Canadian prairies. Prairie Soils Crops 4:114-121. http://www.prairiesoilsandcrops.ca

24. Strelkov, S. E., Manolii, V. P. Hwang, S. F., Howard, R. J. Manolii, A. V., Zhou, Q., Holtz, M., and Yang, Y. 2008. Incidence of clubroot on canola in Alberta in 2007. Can. Plant Dis. Surv. 88:101-102.

25. Strelkov, S. E., Manolii, V. P., Rennie, D. C., Xiao, Q., Cui, D., and Hwang, S. F. 2011. The occurrence of clubroot on canola in Alberta in 2010. Can Plant Dis. Surv. 91:109-111.

26. Strelkov, S. E., Tewari, J. P., and Smith-Degenhardt, E. 2006. Characterization of Plasmodiophora brassicae populations from Alberta, Canada. Can. J. Plant Pathol. 28:467-474.

27. Tewari, J. P., Strelkov, S. E., Orchard, D., Hartman, M., Lange, R. M., and Turkington, T. K. 2005. Identification of clubroot of crucifers on canola (Brassica napus) in Alberta. Can. J. Plant Pathol. 27:143-144.

28. Wallenhammar, A.-C. 1999. Monitoring and control of Plasmodiophora brassicae in spring oilseed brassica crops. Acta Universitatis Agriculturae Sueciae, Agraria 183. Swedish University of Agricultural Sciences, Uppsala, Sweden.

29. Williams, P. H. 1966. A system for the determination of races of Plasmodiophora brassicae that infect cabbage and rutabaga. Phytopathology 56:624 626.

30. Xue, S., Cao, T., Howard, R. J., Hwang, S. F., and Strelkov, S. E. 2008. Isolation and variation in virulence of single-spore isolates of Plasmodiophora brassicae from Canada. Plant Dis. 92:456-462. 